\title{
Concentration of CCL11, CXCL8 and TNF- $\alpha$ in sputum and plasma of patients undergoing asthma or chronic obstructive pulmonary disease exacerbation
}

M.B. Daldegan ${ }^{1}$, M.M. Teixeira ${ }^{1}$ and A. Talvani ${ }^{1,2}$
Correspondence

A. Talvani

Laboratório de Imunofarmacologia Departamento de Bioquímica e

Imunologia, ICB, UFMG

Av. Antônio Carlos, 6627

31270-901 Belo Horizonte, MG

Brasil

Fax: +55-31-3441-5963

E-mail: talvani@pucminas.br

Research supported by FAPEMIG and CNPq.

Received May 21, 2004

Accepted June 1, 2005
1Departamento de Bioquímica e Imunologia, Instituto de Ciências Biológicas, Universidade Federal de Minas Gerais, Belo Horizonte, MG, Brasil

${ }^{2}$ Instituto de Ciências Biológicas e Saúde,

Pontifícia Universidade Católica de Minas Gerais, Belo Horizonte, MG, Brasil

\begin{abstract}
Asthma and chronic obstructive pulmonary disease (COPD) are common respiratory illnesses characterized by chronic inflammation of the airways. The characterization of induced or spontaneously produced sputum is a useful technique to assess airway inflammation. In the present study, we compared the concentrations of CCL2, CCL11, CXCL8, and tumor necrosis factor- $\alpha$ (TNF- $\alpha$ ) in plasma and induced sputum of patients with severe asthma or COPD and correlated the levels of these mediators with inflammatory cells in sputum. Asthmatic patients had elevated levels of eosinophils $(40.1 \pm 6.24 \%)$ in sputum whereas neutrophils $(63.3 \pm 4.66 \%)$ predominated in COPD patients. The levels of the chemokine CCL11 were markedly increased in sputum $(708.7 \pm 330.7 \mathrm{pg} / \mathrm{ml})$ and plasma $(716.6 \pm 162.2$ $\mathrm{pg} / \mathrm{ml}$ ) of asthmatic patients and correlated with the percentage of eosinophils in induced sputum. The concentrations of CXCL8 (817.0 $\pm 105.2 \mathrm{pg} / \mathrm{ml})$ and TNF- $\alpha(308.8 \pm 96.1 \mathrm{pg} / \mathrm{ml})$ were higher in sputum of COPD patients and correlated with the percentage of neutrophils in induced sputum. There was also an increase in the concentrations of CXCL8 $(43.2 \pm 6.8 \mathrm{pg} / \mathrm{ml})$ in sputum of asthmatic patients. These results validate that sputum is a suitable method to assess chemokines and cytokines associated with asthma and COPD. Moreover, the mechanisms involved in the synthesis of CCL11 and CXCL8/TNF- $\alpha$ would be helpful to better understand the inflammatory profile associated with asthma and COPD, respectively.
\end{abstract}

\section{Introduction}

Asthma and chronic obstructive pulmonary disease (COPD) are chronic inflammatory diseases of the airways. Whereas there is a predominance of eosinophils and Th2
Key words - Asthma

- Chronic obstructive pulmonary disease

- Chemokines

- TNF- $\alpha$ cytokine-producing $\mathrm{T}$ cells in the inflammatory infiltrate in the airways of asthmatic patients $(1,2)$, neutrophils predominate in COPD patients (3). As these leukocytes appear to play an important role in disease pathogenesis, it is important that we under- 
stand the molecular signals that drive their influx into the airways if our future goal is to design novel anti-inflammatory strategies.

Chemokines are among the various mediators of the inflammatory process thought to be major mediators of leukocyte differentiation and influx in several disease processes, including asthma and COPD (3-7). Several experimental studies, especially in models of asthma, have shown an important role of chemokines for leukocyte migration into the lungs $(8,9)$. Some studies have demonstrated a positive correlation between CCL11 and eosinophils or eosinophil-derived products in asthmatic individuals (10). There also appears to be a good correlation between CXCL8 and sputum neutrophils in COPD patients (11). Indeed, high levels of CXCL8 are found during an acute exacerbation of COPD and appear to be involved in the development of peripheral muscle weakness (12). In addition, there is an up-regulation of the chemokines CXCL5 and CXCL8 in lung biopsies from patients with severe COPD (7). Thus, there is good justification for the measurement of the concentration of chemokines in the lungs or lung-derived specimens of patients with chronic airway disease.

The main objective of the present study was to evaluate the concentration of chemokines in sputum of patients with severe asthma or undergoing severe COPD exacerbations and to correlate chemokine concentrations with leukocyte influx. We measured the eosinophil-active chemokine CCL11 (eotaxin) and the neutrophil-active chemokines CXCL8 (interleukin-8), CCL3 (macrophage inflammatory protein- $1 \alpha$ ) and CCL2 (monocyte chemoattractant peptide-1), chemokines thought to play an important role in the pathogenesis of chronic airway diseases $(9,13)$. Chemokines were measured in induced or spontaneously produced sputum of patients and control individuals since this technique appears to provide useful overall assessment of inflammation within the air- ways (14). The concentrations of the proinflammatory cytokine tumor necrosis factor- $\alpha$ (TNF- $\alpha$ ) in sputum were also measured for comparison. In addition and in an attempt to evaluate and compare local versus systemic inflammatory events in response to severe airway inflammation, we evaluated the concentrations of chemokines in plasma of asthmatic and COPD patients.

\section{Material and Methods}

\section{Study population}

The study was approved by the ethics review board of Hospital Municipal Odilon Behrens (HMOB), Belo Horizonte, MG, Brazil. All clinical procedures were performed at HMOB or at the Faculdade de Ciências Médicas de Minas Gerais, Belo Horizonte, MG, Brazil, and all individuals gave written informed consent to participate in the study. Patients with a previous diagnosis of asthma or COPD (see below) and undergoing an acute asthmatic attack or COPD exacerbation were recruited at the emergency department of HMOB. Acute exacerbation was defined as an acute worsening of respiratory distress that led patients to seek the emergency department of the hospital. Healthy control individuals $(\mathrm{N}=9)$ were recruited and evaluated clinically at Ambulatório de Fisioterapia Respiratória (Faculdade de Ciências Médicas de Minas Gerais). A complete medical history was obtained for all individuals and all underwent clinical examination and performed the peak expiratory flow procedure.

The diagnosis of asthma was established by clinical and lung function measurements according to the current international guidelines for the diagnosis and treatment of asthma $(15,16)$. Asthmatic individuals $(\mathrm{N}=$ 9) had received no systemic or inhaled corticosteroids during the preceding month. Due to the high cost of medication, the use of inhaled steroids is uncommon among pa- 
tients of lower economic background in Brazil (16). COPD was diagnosed according to the criteria recommended by the European Respiratory Society $(17,18)$. All COPD individuals $(\mathrm{N}=11)$ were ex-smokers and $73 \%$ had been taking orally administered corticosteroids during the month preceding the study.

\section{Collection of plasma and sputum}

Blood was collected into tubes containing heparin (Becton \& Dickinson, Franklin Lakes, NJ, USA) and centrifuged twice at 3,000 $\mathrm{g}$ for $10 \mathrm{~min}$ and the isolated plasma was stored at $-80^{\circ} \mathrm{C}$ until the immunoassays were performed. Total cell counts were performed using an automated system (HemaTek/Milles, Longwood, FL, USA) and differential counts were performed on blood smears stained with May-Grünwald-Giemsa.

Since patients reaching the emergency department were dyspneic, sputum was obtained after micronebulization for $20 \mathrm{~min}$ with bronchodilators. Healthy controls were submitted to micronebulization with saline. After nebulization, individuals were encouraged to cough up sputum on a plastic Petri dish (Becton \& Dickinson) using a huffing technique. Sputum was then collected and separated from saliva and a 1-ml aliquot was stored at $-80^{\circ} \mathrm{C}$. The remaining material was homogenized in the presence of dithiothreitol (DTT, 1:1) (19) and incubated in a shaking water bath for $20 \mathrm{~min}$ at $37^{\circ} \mathrm{C}$. After centrifugation, the supernatant was discarded and the pellet resuspended in a phosphatebuffered saline (PBS) solution containing $3 \%$ bovine serum albumin (BSA). Differential cell counts were performed on cytospin preparations (Shandon III, Pittsburgh, PA, USA) stained with May-Grünwald-Giemsa using standard morphological criteria to identify cell types. The results are reported as percentage of each cell type in $10 \mu \mathrm{l}$ of sputum. All sputum samples contained less than $20 \%$ epithelial cells.

\section{Measurement of TNF- $\alpha$ and chemokines in plasma and sputum}

Human detection kits of TNF- $\alpha$ and chemokines CCL2 (monocyte chemoattractant peptide-1), CCL3 (macrophage inflammatory protein-1 $\alpha$ ), and CCL11 (eotaxin) were from Pharmingen (San Diego, CA, USA), and CXCL8 (interleukin-8) from Peprotech (Rocky Hill, NJ, USA). For the measurement of these soluble proteins in plasma, samples were thawed, diluted 1:3 in the appropriate buffer and used for protein determination by ELISA using commercially available antibodies and according to the supplier's protocol. For the evaluation of TNF- $\alpha$ and chemokines in sputum, samples were thawed and centrifuged at $15,000 \mathrm{~g}$ for $1 \mathrm{~h}$ and the supernatant was diluted 1:3 in $0.1 \%$ PBS-BSA (Sigma, St. Louis, MO, USA).

\section{Statistical analysis}

For comparison between different groups, parametric and non-parametric data were compared by analysis of variance (ANOVA) followed by Student-Newman-Keuls' or Dun's post hoc analysis, respectively. For association between CCL11/CXCL8 and TNF- $\alpha$ expression and cellular pattern, data were analyzed using linear regression analysis and Spearman's rank correlation test. Probability values were considered significant when $\mathrm{P}<0.05$. Data are reported as means \pm SEM or as the median and confidence interval (non-parametric). Analysis was performed using the computer program PRISM (GraphPad, San Diego, CA, USA).

\section{Results}

The age distribution of healthy individuals and patients with asthma or COPD is shown in Table 1 . The mean age of asthmatic patients was similar to that of control individuals, while patients with COPD were 
significantly older. The severity of the disease for both asthma and COPD patients was demonstrated by the low percentage of peak expiratory flow rates and, in asthmatic patients, by the significant increase in respiratory rate (Table 1 ).

Eosinophils comprised approximately $40 \%$ of the infiltrating leukocytes in sputum from asthmatic patients (Table 2). In contrast, the majority of infiltrating leukocytes in sputum from COPD patients were neutrophils. Asthmatic patients also had a higher percentage of neutrophils than healthy controls.

The concentration of chemokines in sputum and plasma from healthy individuals and patients with asthma or COPD is shown in Figure 1. Sputum and plasma CCL11 concentrations were higher in individuals

Table 1. Clinical parameters of individuals with asthma and chronic obstructive pulmonary disease (COPD).

\begin{tabular}{lccc}
\hline & Healthy controls $(\mathrm{N}=9)$ & Asthma $(\mathrm{N}=9)$ & COPD $(\mathrm{N}=11)$ \\
\hline Age & $37 \pm 4.9$ & $37 \pm 6.1^{+}$ & $62 \pm 3.6^{*}$ \\
Sex $(\%$ male $)$ & 22.2 & 44.4 & 54.5 \\
PF\% & $94.8 \pm 1.8$ & $43.3 \pm 6.1^{*}$ & $52.0 \pm 5.3^{*}$ \\
RF & $18.2 \pm 0.6$ & $25.3 \pm 2.7^{*+}$ & $18.4 \pm 3.9$ \\
\hline
\end{tabular}

Data are reported as means \pm SD. PF\% = peak of respiratory flow; RF = respiratory frequency.

${ }^{*} \mathrm{P}<0.01$ compared to healthy controls and ${ }^{+} \mathrm{P}<0.01$ compared to COPD (ANOVA and Student-Newman-Keuls test).

Table 2. Inflammatory cells in sputum of patients with asthma and chronic obstructive pulmonary disease (COPD).

\begin{tabular}{lccr}
\hline & Healthy controls $(\mathrm{N}=9)$ & Asthma $(\mathrm{N}=9)$ & COPD $(\mathrm{N}=11)$ \\
\hline Eosinophils & - & $40.06 \pm 6.24^{*+}$ & $5.14 \pm 1.03$ \\
Neutrophils & $5.22 \pm 1.23$ & $18.57 \pm 4.12^{+}$ & $63.32 \pm 4.66^{*}$ \\
\hline
\end{tabular}

Data are reported as means \pm SD in percentage. Differential cell counts were carried out on sputum treated with dithiothreitol in cytospin preparations stained with MayGrünwald-Giemsa using standard morphological criteria to identify eosinophils and neutrophils.

${ }^{*} \mathrm{P}<0.001$ compared to healthy controls and ${ }^{+} \mathrm{P}<0.001$ compared to COPD (ANOVA and Student-Newman-Keuls test). with asthma than in those with COPD or with no respiratory disease (Figure 1A and B). In contrast, sputum and plasma CXCL8 concentrations were higher in COPD patients than in patients with asthma or healthy control individuals (Figure 1C and D). Of note, patients with acute severe asthma had higher concentrations of CXCL8 in sputum, but not in plasma, when compared with healthy control individuals (Figure 1C and D). There was no significant difference in the concentrations of CCL2 in sputum or plasma from individuals with asthma or COPD or from healthy control individuals (Figure $1 \mathrm{E}$ and $\mathrm{F}$ ). The concentrations of TNF- $\alpha$ in sputum of patients with COPD were higher than those of patients with asthma or control healthy individuals (Figure 2A) while its concentration in plasma was not different between asthma and COPD patients (data not shown). The chemokine CCL3 was detected only in sputum (Figure 2B), but not in plasma (data not shown) and there did not appear to be any difference between the asthma and COPD groups.

Finally, we determined whether the concentrations of these chemokines correlated with the type of leukocytes present in patient sputum. There was a significant positive correlation between the sputum concentrations of TNF- $\alpha(r=0.66, P=0.0013)$ or CXCL8 ( $r=0.74, P=0.0002)$ and the number of neutrophils in sputum of patients with COPD and asthma. Similarly, there was a positive correlation between the levels of CCL11 and the number of eosinophils $(r=$ $0.60, \mathrm{P}=0.005$ ) in sputum of patients. We also found a significant correlation between the plasma and sputum concentrations of CCL11 in patients with asthma $(r=0.82$, $\mathrm{P}=0.006$ ) but this correlation was not significant when CXCL8 was compared in the same group of patients $(\mathrm{r}=0.12, \mathrm{P}=$ 0.71 ). Similarly, there was no positive correlation between CXCL8 levels in plasma and serum of patients with COPD $(\mathrm{r}=0.25, \mathrm{P}=$ $0.27)$. 

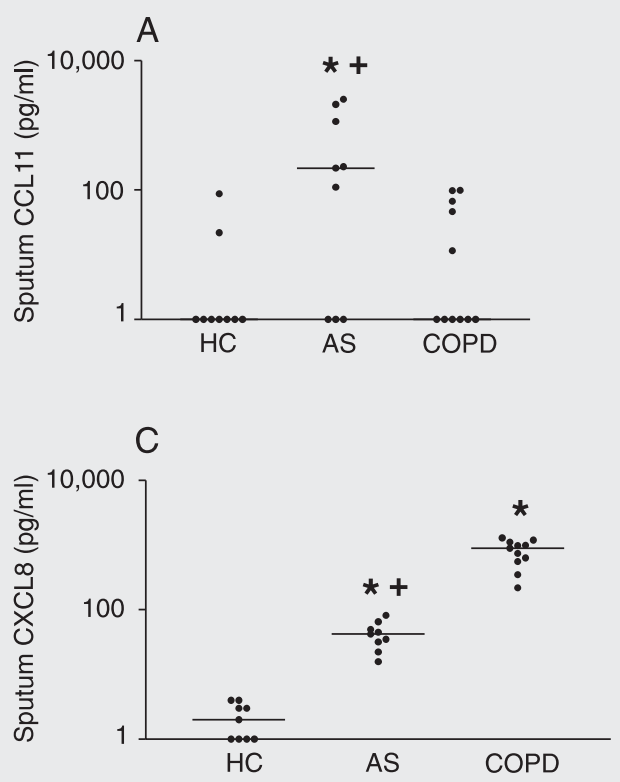

E

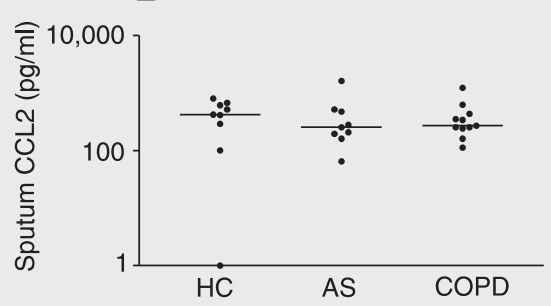

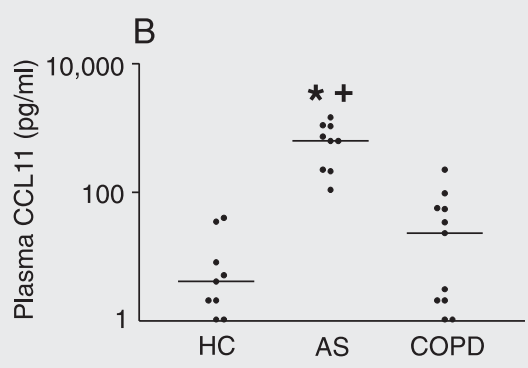
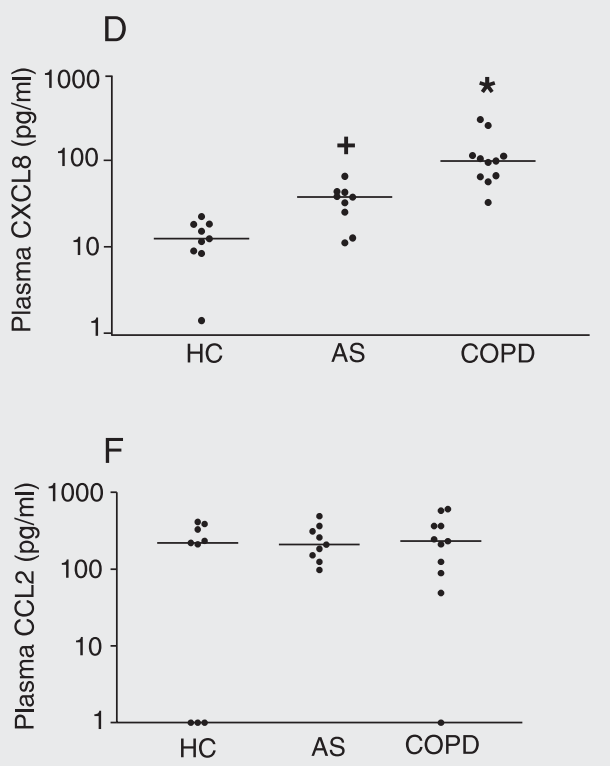

\section{Discussion}

Analysis of sputum is a useful strategy to identify inflammatory mediators associated with relevant pathophysiological mechanisms of airway respiratory diseases $(14,20)$. There is some disagreement between investigators regarding the effect of mucolytic agents such as DTT on the measurement of some inflammatory mediators (20). However, in our experiments with untreated and DTT-treated sputum there were no differences in these cytokine concentrations (data not shown). In the present study, we evaluated the concentrations of the chemokines CCL2, CCL3, CCL11, and CXCL8 in sputum of patients with asthma or COPD under-

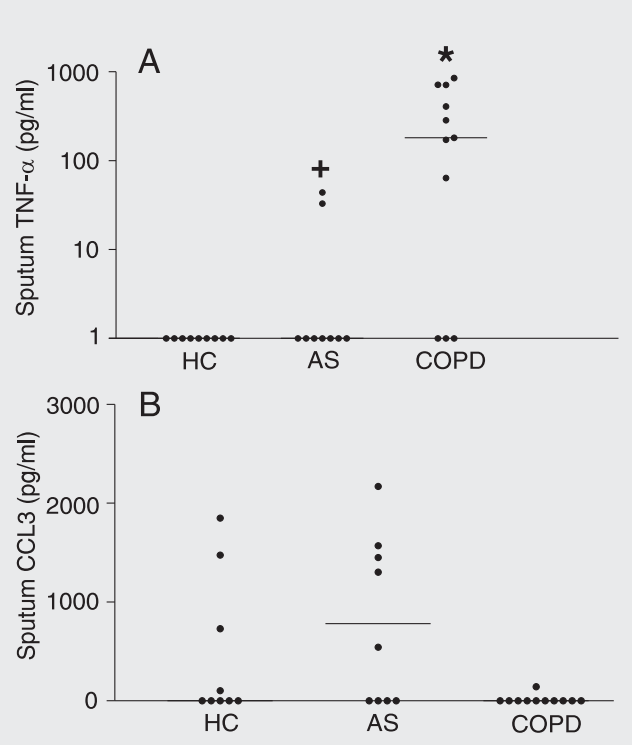

Figure 1. Concentrations of chemokines in sputum (left side) and plasma (right side) of healthy control $(\mathrm{HC}, \mathrm{N}=9)$ and patients with asthma (AS, N = 9) or chronic obstructive pulmonary disease (COPD, $N=11$ ). The concentration of CCL11 (A, B), CXCL8 (C, D) or CCL2 (E, F) in sputum or plasma was measured by ELISA. Data are reported as the log mean value. The horizontal lines indicate the median value for each group. ${ }^{*} \mathrm{P}$ $<0.05$ compared to $\mathrm{HC}$ and $+P$ $<0.05$ compared to COPD (Spearman's rank correlation test).

Figure 2. Concentrations of TNF- $\alpha$ and CCL3 in sputum of healthy controls $(\mathrm{HC}, \mathrm{N}=9)$ and patients with asthma (AS, N = 9) or chronic obstructive pulmonary disease (COPD, $N=11$ ). The concentration of TNF- $\alpha$ (A) and $\mathrm{CCL} 3(\mathrm{~B})$ in sputum was measured by ELISA. Data are reported as the log mean value. The horizontal lines indicate the median value for each group. ${ }^{*} \mathrm{P}$ $<0.05$ compared to $\mathrm{HC}$ and $+\mathrm{P}$ $<0.05$ compared to COPD (Spearman's rank correlation test). 
going a clinical exacerbation. These were patients with very severe disease in whom the determination of chemokine levels has not been reported. For comparison, the levels of these chemokines were also measured in plasma.

Studies measuring chemokines in sputum from asthmatic patients have shown elevated concentrations of CCL11 (eotaxin) and a high number of eosinophils $(9,10$, 21,22). Here, we evaluated asthmatic patients during an acute exacerbation of asthma. Of interest, none of our patients were using steroids in the month preceding the study. In Brazil, drugs recommended for the treatment of asthma are not available via the governmental health system (16). For this reason and due to the high cost of these medications, the majority of patients fail to obtain adequate medical treatment. This is a unique opportunity to evaluate changes in the lung of steroidal treatment-naive patients. In these patients, we found a high percentage of eosinophils and a high concentration of CCL11 in sputum and plasma. The levels of CCL2 and CCL3 were not different in the sputum of patients with asthma or normal individuals. Importantly, CCL11 correlated with the percentage of eosinophils in sputum and there was also a positive correlation between plasma and sputum levels of CCL11.

Overall, our results reinforce findings of other studies $(10,22)$ and suggest that CCL11 may be important for eosinophil recruitment into the lungs of patients with acute asthmatic exacerbation. The demonstration that CCL11 is elevated in plasma may also be relevant regarding the ability of CCL11 to recruit eosinophils from bone marrow (23). More importantly, these results suggest that plasma chemokine levels are good markers of the inflammatory events occurring in the lungs of these patients with an acute exacerbation of asthma. Future studies will be necessary to define whether CCL11 is directly associated with changes in airway function and whether measuring CCL11 in plasma will help define the prognosis of patients.

Neutrophils have been suggested to play a role during the acute exacerbation of asthma (24). Interestingly and in addition to CCL11, patients with asthma had elevated levels of CXCL8 and neutrophils in sputum and there was a positive correlation between these two variables. This is consistent with the results of other studies demonstrating the presence of neutrophils and CXCL8 during acute exacerbation of asthma (25-27). Nevertheless, there was no close relationship between sputum and plasma CXCL8 levels, suggesting that measurement of this chemokine in plasma is not useful to infer changes in the lung. Some patients were taking orally administered corticosteroids during the month preceding sample collection. But the real differences observed in the levels of CCL11 and eosinophils might agree with data obtained in previous experiments using a dexamethasone model in guinea pigs which suggested that corticosteroids suppress eosinophil accumulation in the lung tissue and airways but have no significant effect on eotaxin levels (28).

Several studies have demonstrated that the concentrations of TNF- $\alpha$ and CXCL8 are elevated in sputum of patients with COPD (3-7). Our results are consistent with these findings and demonstrate further that CXCL8 is also elevated in plasma of COPD patients. There was a good correlation between concentrations of TNF- $\alpha$ and CXCL 8 and the presence of neutrophils in sputum, suggesting that these mediators may participate in the cascade of events leading to neutrophil influx and neutrophil-mediated damage. In contrast, we did not detect elevated concentrations of CCL2, CCL3 or CCL11. The absence of elevated levels of CCL11 in sputum or plasma of these patients suggests that measurement of this chemokine may be helpful to define the differential diagnosis between these two conditions, although further studies are necessary to confirm the latter statement. 


\section{Acknowledgments}

We are grateful to Dr. Gilberto Jose Mar- tins, Hospital Municipal Odilon Behrens, Belo Horizonte, MG, Brazil, for performing the blood biochemistry analysis.

\section{References}

1. Holgate S (1993). Mediator and cytokine mechanism in asthma. Thorax, 48: 103-109.

2. Bettiol J, Sale J, Henket M et al. (2002). Cytokine production from sputum cells after allergenic challenge in IgE-mediated asthma. Allergy, 57: 1145-1150.

3. Saetta M, Baraldo S \& Zuin R (2003). Neutrophil chemokines in severe exacerbations of chronic obstructive pulmonary disease. American Journal of Respiratory and Critical Care Medicine, 168: 911-913.

4. Robinson DS (2000). Th-2 cytokines in allergic disease. British Medical Bulletin, 56: 956-968.

5. Boer WI (2002). Cytokines and therapy in COPD. Chest, 121: 209s$218 \mathrm{~s}$.

6. Lamkhioued B, Abdelilah SG, Hamid Q et al. (2002). The CCR3 receptor is involved in eosinophil differentiation and is up-regulated by Th2 cytokines in CD34 progenitor cells. Journal of Immunology, 169: $537-547$.

7. Qiu Y, Zhu J, Bandi V et al. (2003). Biopsy neutrophilia, neutrophil chemokine and receptor gene expression in severe exacerbations of chronic obstructive pulmonary disease. American Journal of Respiratory and Critical Care Medicine, 168: 968-975.

8. Mould AW, Matthaei KI, Young IG et al. (1997). Relationship between interleukin-5 and eotaxin in regulating blood and tissue eosinophilia in mice. Journal of Clinical Investigation, 99: 1064-1070.

9. Panina-Bordignon $P$ \& D'Ambrosio D (2000). Chemokines and their receptors in asthma and chronic obstructive pulmonary disease. Current Opinion in Pulmonary Medicine, 9: 104-110.

10. Jahnz-Ro K, Plusa T \& Mierzejewska J (2000). Eotaxin in serum of patients with asthma or chronic obstructive pulmonary disease: relationship with eosinophil cationic protein and lung function. Mediators of Inflammation, 9: 175-179.

11. Yamamoto C, Yoneda T, Yoshikawa M et al. (1997). Airway inflammation in COPD assessed by sputum levels of interleukin-8. Chest, 112: $505-510$

12. Spruit MA, Gosselink R, Troosters T et al. (2003). Muscle force during an acute exacerbation in hospitalized patients with COPD and its relationship with CXCL8 and IgG-I. Thorax, 58: 741-742.

13. Owen C (2001). Chemokine receptors in airway disease: which receptors to target? Pulmonary Pharmacology and Therapeutics, 14: 193-202.

14. Out TA, Jansen HM \& Lutter R (2001). Methodological aspects in the analysis of spontaneously produced sputum. Monaldi Archives for Chest Disease, 56: 493-499.

15. Sheffer AL (1990). Global Initiative for Asthma. NHLBI/WHO Report. $\mathrm{NIH}$, Bethesda, MD, USA, Suppl I, 2-8.
16. Anonymous (2002). III Consenso Brasileiro no Manejo da Asma. Jornal de Pneumologia, 28 (Suppl 1): S3-S28.

17. Siafakas NM, Vermeire P, Pride NB et al. (1995). Optimal assessment and management of chronic obstructive pulmonary disease (COPD). European Respiratory Journal, 8: 1398-1420.

18. Anonymous (2000). I Consenso Brasileiro de Doença Pulmonar Obstrutiva Crônica. Jornal de Pneumologia, 26 (Suppl 1): S1-S52.

19. Bresser $P$, Out $T A$, van Alphen $L$ et al. (2000). Airway inflammation in nonobstructive and obstructive chronic bronchitis with chronic Haemophilus influenze airway infection. Comparison with noninfected patients with chronic obstructive pulmonary disease. American Journal of Respiratory and Critical Care Medicine, 162: 947952.

20. Woolhouse IS, Bayley DL \& Stockley RA (2002). Effect of sputum processing with dithiothreitol on the detection of inflammatory mediators in chronic bronchitis and bronchiectasis. Thorax, 57: 667671.

21. Stankiewicz W, Dabrowski MP, Chcialowski A et al. (2002). Cellular and cytokine immunoregulation in patients with chronic obstructive pulmonary disease and bronchial asthma. Mediators of Inflammation, 11: 307-312.

22. Park SW, Kim J, Chang HS et al. (2003). Association of interleukin5 and eotaxin with acute exacerbation of asthma. International Archives of Allergy and Immunology, 131: 283-290.

23. Palframan RT, Collins PD, Severs NJ et al. (1988). Mechanisms of acute eosinophil mobilization from the bone marrow stimulated by interleukin-5: The role of specific adhesion molecules and phosphatidylinositol 3-kinase. Journal of Experimental Medicine, 188: 16211632.

24. Ennis M (2003). Neutrophils in asthma pathophysiology. Current Allergy and Asthma Reports, 3: 159-165.

25. Gordon JR, Swystun VA, Li F et al. (2003). Regular salbutamol use increases CXCL8 responses in asthma: relationship to the eosinophil response. European Respiratory Journal, 22: 118-126.

26. Persson T, Monsef N, Andersson P et al. (2003). Expression of the neutrophil-activating CXC chemokine ENA-78/CXCL5 by human eosinophils. Clinical and Experimental Allergy, 33: 531-537.

27. Chanez P, Enander I, Jones I et al. (1996). Interleukin 8 in bronchoalveolar lavage of asthmatic and chronic bronchitis patients. International Archives of Allergy and Immunology, 111: 83-88.

28. Humbles AA, Conroy DM, Marleau S et al. (1997). Kinetics of eotaxin generation and its relationship to eosinophil accumulation in allergic airways disease: analysis in a guinea pig model in vivo. Journal of Experimental Medicine, 186: 601-612. 